This is an electronic reprint of the original article. This reprint may differ from the original in pagination and typographic detail.

Author(s): Finni Juutinen, Taija; Ikegawa, Shikegi; Komi, Paavo

Title: Concentric force enhancement during human movement

Year: $\quad 2001$

Version:

Please cite the original version:

Finni Juutinen, T., Ikegawa, S., \& Komi, P. (2001). Concentric force enhancement during human movement. Acta Physiologica Scandinavica, 2001(173), 369-377.

All material supplied via JYX is protected by copyright and other intellectual property rights, and duplication or sale of all or part of any of the repository collections is not permitted, except that material may be duplicated by you for your research use or educational purposes in electronic or print form. You must obtain permission for any other use. Electronic or print copies may not be offered, whether for sale or otherwise to anyone who is not an authorised user. 


\section{CONCENTRIC FORCE ENHANCEMENT DURING HUMAN MOVEMENT}

Taija Finni ${ }^{1}$, Shigeki Ikegawa ${ }^{2}$ and Paavo V. Komi ${ }^{1}$

${ }^{1}$ Neuromuscular Research Center, Department of Biology of Physical Activity, University of Jyväskylä, Jyväskylä, Finland

${ }^{2}$ Laboratory for Exercise Physiology, Tokyo Metropolitan College, Tokyo, Japan

$\triangle$ Taija Finni

Neuromuscular Research Center

Department of Biology of Physical Activity

University of Jyväskylä

P.O. Box 35 (LL 227), 40351 Jyväskylä, Finland

Tel: (+) 358405566582

Fax: (+) 358142602071

E-mail: finni@maila.jyu.fi

Running title: Concentric force enhancement 


\begin{abstract}
In order to understand the possible mechanisms contributing to enhanced concentric performance in stretch-shortening cycle exercises in vivo the present study examined knee extension torque, EMG activity and fascicle length of the vastus lateralis muscle in maximal and submaximal human movements. Maximal concentric knee extensions $\left(120^{\circ} \mathrm{s}^{-1}\right)$ were done after pre-stretch and pre-isometric conditions by nine volunteers. During shortening at the knee angle of $115^{\circ}\left(180^{\circ}=\right.$ extended $)$ the knee extension torque was found to be greater in pre-stretch condition (272 vs. $248 \mathrm{Nm}, p<0.05)$ although the torque level prior to shortening was smaller than in pre-isometric condition (268 vs. $314 \mathrm{Nm}, p<0.05)$. At the moment of torque enhancement the EMG activity levels or fascicle lengths did not differ between the conditions. It is proposed that besides specific experimental conditions the present enhancement may be related to longer fascicle length prior to shortening (by $4.1 \mathrm{~cm}, p<0.05$ ) in prestretch condition and to modified length-tension properties. Fascicle length behaviour was found to play an important role also in unilateral, submaximal sledge-jump conditions where pre-loading was altered but the concentric net impulse and joint angular movements were the same. In repeated drop jumps with greater pre-load the changes in fascicle length were smaller than in the counter movement jump that was characterized by a lower force and activity level in the eccentric phase. Results from the present maximal and submaximal loading conditions suggest that the benefits of stretch-shortening cycle muscle function may come through different interactive mechanisms that may be task specific.
\end{abstract}

Key words: fascicle, muscle-tendon unit, force enhancement, potentiation, force-length, stretch-shortening cycle, knee extension, jumping

\title{
Introduction
}

Stretch-shortening cycle (SSC) muscle function is commonly utilized in our everyday locomotion. In SSC an active muscle is stretched prior to shortening (Norman \& Komi 1979). In human movement studies, however, the term is often used without information about the change in muscle length; only a muscle-tendon unit is observed to lengthen prior to shortening. In fact, animal experiments have shown that changes in muscle tendon unit length do not always correspond to changes in the muscle fibres themselves during natural locomotion (e.g. Roberts et al. 1997). The dominant role of SSC muscle action stems from performance potentiation that deals with such concepts as 1) utilization of elastic energy (Aura \& Komi 1986, Bosco et al. 1982, Komi 1983), 2) activation mechanics (Bobbert et al. 1996) with reflex contribution (Bosco et al. 1982, Gollhofer et al. 1992, Komi \& Gollhofer 1997) and 3) history dependence of force production (Herzog \& Leonard 2000). 
Although the true nature of SSC exercise is difficult to assess using isolated animal models with stimulation input, they have provided fundamental knowledge of muscle mechanics. Especially, the stretch-induced enhancement of force is a well-known phenomenon from animal studies (e.g. Cavagna et al. 1968, Edman et al. 1978). A stretch-induced contractile potentiation (with transient and residual components) during shortening has been shown to occur and authors have suggested that it could be a potential mechanism to act in stretch-shortening cycle (SSC) exercise in vivo (Cavagna et al. 1968, Ettema et al. 1992). Although the residual force enhancement component has been proposed to have relevance in vivo (Edman 1997), recent observations by Brown \& Loeb (2000) suggest negligible effects from contractile potentiation during in vivo locomotion. In fact, many human studies have stressed the importance of elastic recoil as a source for potentiation together with higher activation (Asmussen \& Bonde-Petersen 1974, Bosco et al. 1981, 1982).

Typically, maximal activation or effort has been used in protocols focusing on different aspects of performance enhancement. In normal locomotion, however, the intact neuromuscular system can produce constantly varying activity levels. In comparison of drop jump (DJ) and counter movement jumps $(\mathrm{CMJ})$, for example, the lengthening of the muscle occurs with considerable activity or with minor activity, respectively (Gollhofer et al. 1992). Consequently, the pre-loading phase prior to the concentric movement is different and can have an effect on the mechanisms operative in performance potentiation.

Using maximal knee extension, it has been demonstrated that the concentric force production is enhanced when the concentric action is preceded by a stretch (Komi 1983). In these experiments, however, the force level from which the concentric phase began was higher in the pre-stretch than in the pure concentric condition. A high level of force prior to the concentric phase may play a role in performance enhancement also when the squatting (SJ) and counter movement jumps (CMJ) are compared (Bobbert et al. 1996). However, the conditions inside the muscle that may influence potentiation, such as muscle length, velocity, force level and activity patterns, vary in different performances. Because of variable movement strategies and muscle function, the benefits of SSC exercise may appear through different mechanisms and one explanation cannot be generalized to cover all tasks.

In order to understand the potentiating effect of pre-stretch movement in human locomotion, both maximal and submaximal conditions were used in the present experiments. First, maximal concentric force production was compared in pre-stretch (SSC) and pre-isometric conditions (ISO). Our aim was to eliminate the effect of unequal pre-force levels prior to concentric action and to examine if potentiation still could be observed. As we hypothesized that there would be potentiation, the effects of activation and fascicle and muscle-tendon unit lengths on the force production were monitored. In addition to maximal knee extension, we chose to measure submaximal jumping performances that were performed in two controlled conditions where the concentric phases were 
identical in terms of joint angular changes and produced concentric net impulse, but the pre-loading phase was different in terms of muscular activity and force. Thus, we were able to evaluate the effects the different activity level and load in the pre-stretch phase had on the following concentric phase.

\section{Methods}

Nine volunteers participated in this study ( 7 males, 2 females, age $27 \pm 4$ years, height $180 \pm 7 \mathrm{~cm}$, body mass $75 \pm 8 \mathrm{~kg}$ ). They were informed of all the risks associated with the study and they gave their written consent to participate. Recommendations contained in the Declaration of Helsinki were followed and the Ethics Committee of the Central Hospital of Central Finland approved the study.

\section{Knee extension measurements}

First, the subjects were seated in a leg extension ergometer (Komi et al. 2000) where parallel alignment of the rotation axis of a lever arm and knee joint were tested with passive and active extension-flexion movements. The lower leg of the subject was fixed to the lever arm of the ergometer that was equipped with a strain gauge transducer and lever arm length could be read from an inbuilt ruler. Angular displacement of the lever arm was measured with a potentiometer.

First, maximal voluntary contractions at knee angles of $90^{\circ}, 120^{\circ}, 140^{\circ}$ and $160^{\circ}$ were measured (180 corresponds to full knee extension). Then, unilateral knee extensions were performed with isometric (ISO) and eccentric preloading (SSC) (Fig. 1). The range of knee angular motion was $70^{\circ}$ and the velocity of the ergometer lever arm was $120{ }^{\circ} \mathrm{s}^{-1}$. Constant speed of the lever arm was reached within $50 \mathrm{~ms}$ and maintained until the lever arm reached a magnetic stop. Due to the properties of the magnetic stop, a delay of $80 \mathrm{~ms}$ was introduced between the eccentric and concentric phases in SSC. During the delay the lever arm did not move. In ISO the subjects were asked to extend their knee maximally in the isometric preloading phase and to sustain the maximum effort throughout the concentric movement. In SSC the subjects were asked to resist maximally the forced lengthening of the quadriceps femoris muscle (QF) when the lever arm of the ergometer moved from a knee angle of $160^{\circ}$ to $90^{\circ}$. In the subsequent concentric phase the maximum effort was sustained throughout the movement. 

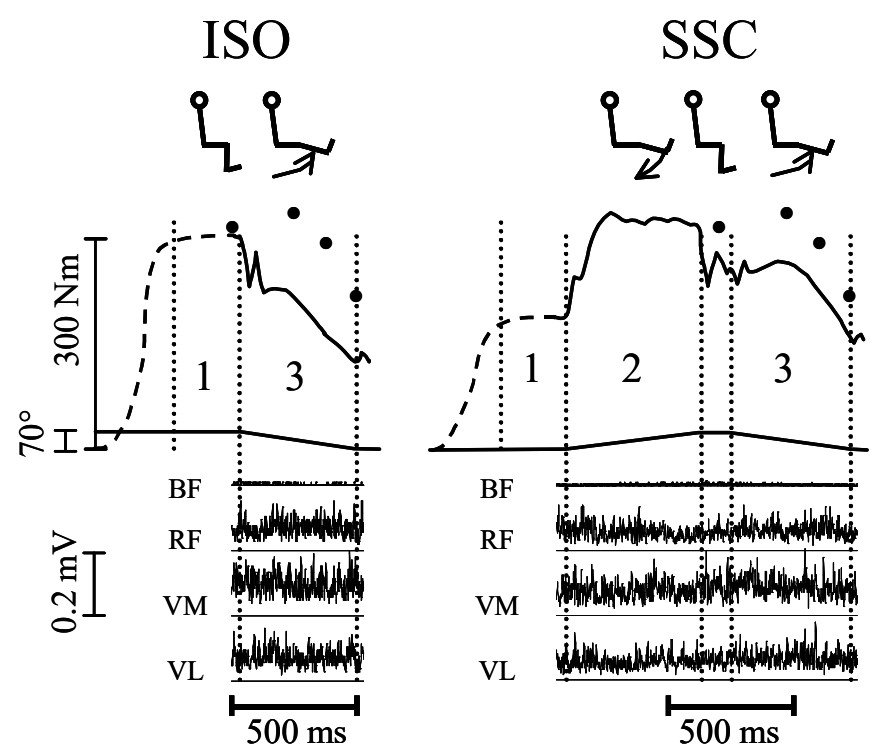

FIGURE 1 Experimental conditions in the maximal knee extension with isometricconcentric (ISO) and eccentric-concentric (SSC) muscle action. Average torquetime curves are shown in the upper curve, divided into different phases according to the changes in the knee joint angle (2. curve from top). Numbered phases between vertical lines correspond to 1) maximal isometric loading (note: different starting angles in ISO and SSC), 2) lengthening and 3) shortening of quadriceps femoris muscles. The delay between the phases 2 and 3 in SSC is caused by the properties of the device. The shortening phase (3) was divided into $50 \mathrm{~ms}$ phases and average force and EMG values were calculated during those periods. Rectified EMG recordings $(n=9)$ from biceps femoris $(B F)$, rectus femoris (RF), vastus medialis (VM) and vastus lateralis (VL) showed no marked differences during the concentric phase. The initial torque rise is dashed schematically in the figure. The group mean EMG recordings for this period could not be obtained. The filled circles represent mean torques measured during maximal voluntary contraction at the given knee angle.

Sledge measurements

In the sledge apparatus (Kyröläinen \& Komi 1995) the subjects performed unilateral CMJs and repeated drop jumps (REP) (Figs. 2, 3). The inclination of the sledge was $24.6^{\circ}$ from horizontal position. The movement of the sledge seat $(30 \mathrm{~kg})$ and therefore also the joint angular changes were predetermined and kept the same in both conditions. The target height was set at $125 \%$ of the toe standing height of each individual subject. Visual feedback of the movement of the sledge seat enabled the subjects to repeat their performances with very little variation. In these jumps the coefficient of variation ranged between 3 and $6 \%$.

In sledge jumps the objective was to examine changes in EMG activity and fascicle length induced by different impact loads in REP and CMJ. Knee angular displacement and concentric net impulse was the same $(p<0.05)$ in both jumps. At least five jumps from each subject were averaged. Group mean curves presented in the Results are time normalized. In sledge jumps the 
downward and upward movements do not correspond exactly with eccentric and concentric phases in the vastus lateralis muscle. Consequently, the terms braking and push-off phase are used when downward and upward movements are considered, respectively (phases 2 and 3 in Fig. 2).

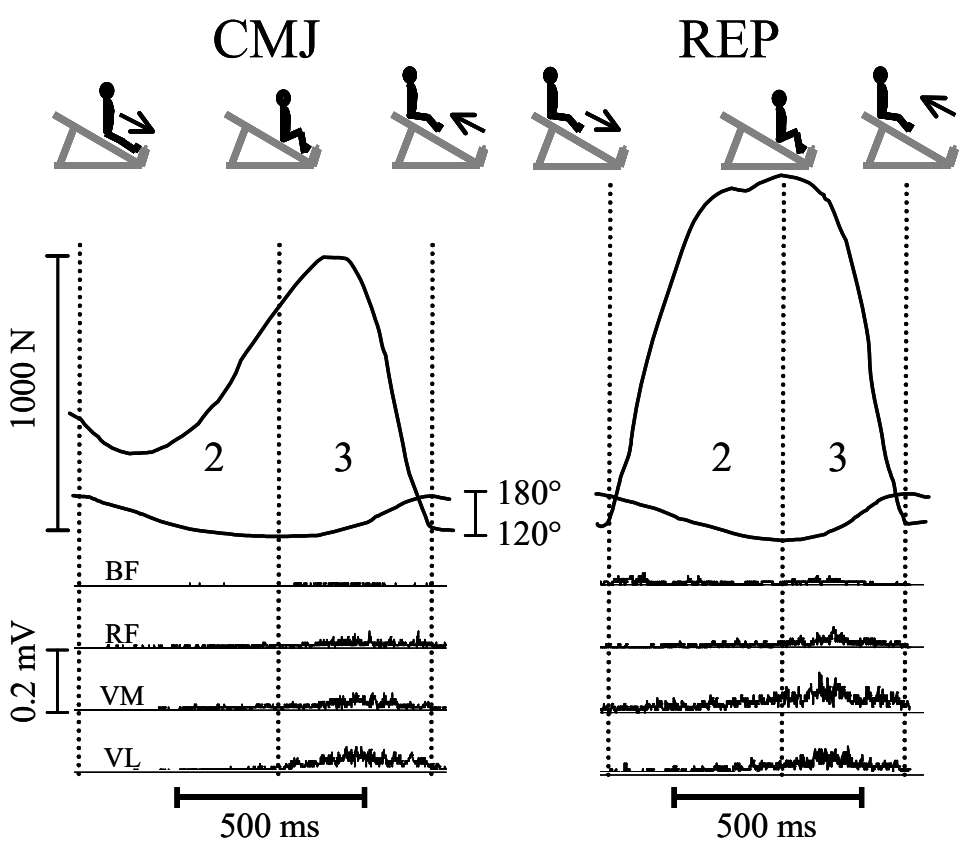

FIGURE 2 Reaction force (upper), knee joint angle (middle) and rectified EMG recordings during submaximal, unilateral counter movement jump (CMJ) and repeated drop jump (REP). For other definitions, see Fig. 1.

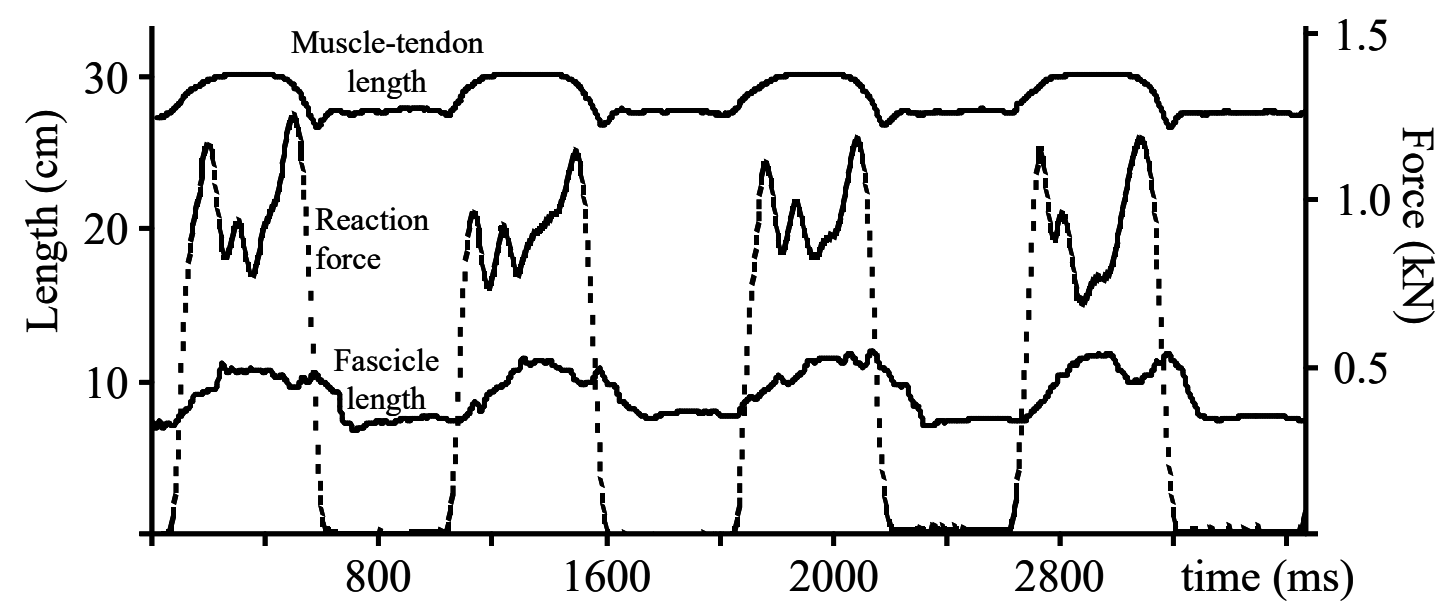

FIGURE 3 An example of raw data recordings from repeated drop jumps. The curves show changes in vastus lateralis muscle-tendon length (upper), fascicle length (lowest curve) and reaction force (dashed line).

\section{Recorded signals}

Extension force and reaction force $(\mathrm{Fz}$, perpendicular to the movement plane of the sledge seat) were measured in the knee extension and sledge performances, 
respectively. Inertial effects of the knee extension machine were minimized by subtracting the strain gauge signal measured in passive condition from that obtained in the active effort. The values were then converted to torques using the lever arm measure, and averaged separately in the different phases (see Fig. 1 and 2). By comparing the output at corresponding knee angles, i.e. with the same lever arm lengths, the force output should be proportional to the produced torque. Bipolar EMG electrodes with an interelectrode distance of 20 mm (Beckmann miniature skin electrodes, USA) were placed between the motor point and the insertion point of distal tendon on the vastus lateralis (VL), rectus femoris, vastus medialis and biceps femoris muscles. The EMG signals were amplified and sent telemetrically to the recording computer. The signals were high-pass filtered $(20 \mathrm{~Hz}$, before sampling), full-wave rectified, integrated and averaged separately in different phases of the movements. In addition to the average values in the entire concentric / push-off phase, the movement was divided into $50 \mathrm{~ms}$ periods. The average torque and average EMG (aEMG) values were obtained from those periods as well. In knee extension the preconcentric torque was defined as an average value for a $50 \mathrm{~ms}$ period just prior to the onset of the concentric phase. The corresponding $50 \mathrm{~ms}$ average values were compared between experimental conditions. Forces and EMGs were collected to a PC with a sampling frequency of $1000 \mathrm{~Hz}$.

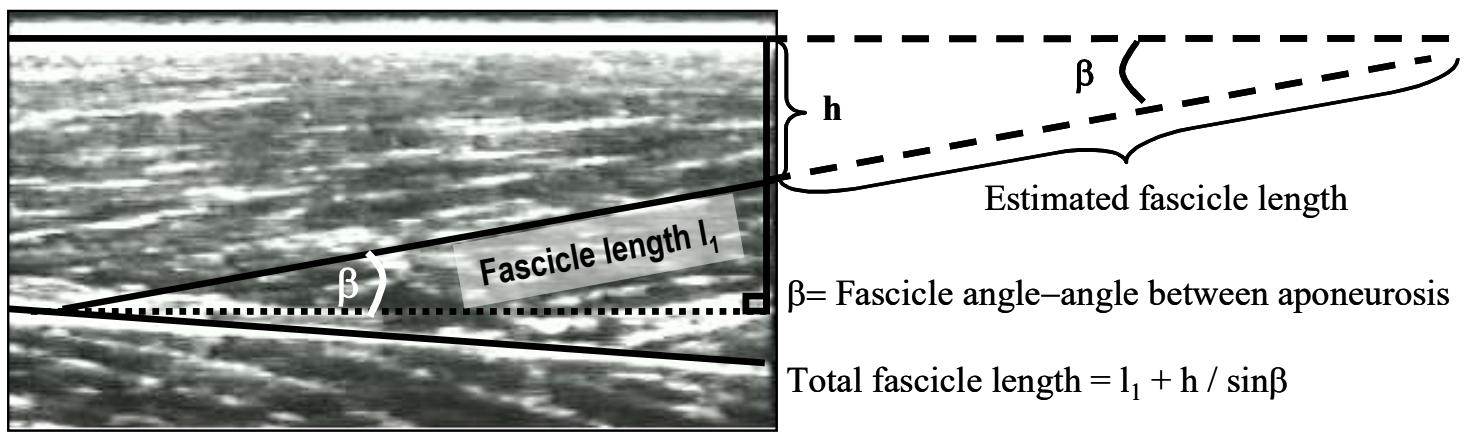

FIGURE 4 The model used for fascicle length determination. The fascicle length $\left(l_{1}\right)$ measured from the image was added to the estimated length $\left[\mathrm{h}(\sin \beta)^{-1}\right]$ to obtain the total fascicle length. The ending points of the chosen $l_{1}$ were tracked throughout the motion. In the model the spatial coordinates of the ending points of the measure $h$ are fixed horizontally to the right end of the visualized area but not in respect to its vertical position or to its position in respect to the fascicle.

Vastus lateralis fascicle lengths were imaged by an Aloka SSD-2000 ultrasonographic device, similarly as reported previously (Ichinose et al. 1997). Images obtained from mid-thigh with a scanning frequency of $42 \mathrm{~Hz}$ were recorded on a videotape at $50 \mathrm{~Hz}$. After a clear image of the echoes from VL fascicle interspaces was visually confirmed by a researcher, a linear array probe $(8 \mathrm{~cm}, 7.5 \mathrm{MHz})$ was firmly secured to subject's thigh with a special support device. The visibility of echoes during contraction and movement was also tested carefully. A parallelogram model as defined by Finni et al. (2001) (Fig. 4) 
was used when the video image was digitised with Motus software (Peak Performance Technologies, USA.). The method for estimating the fascicle length contains an error of 2-7 \% due to curvature of the aponeurosis and fascicles. This error was measured from the printed images of the entire VL muscle. They were constructed from recordings of adjacent images collected along the muscle length. The error is minimum at the level of mid-thigh from where the given fascicle was chosen for analysis. The fascicle had to be clearly visible within the image area throughout the given performance. The same fascicle was followed throughout the motion. It must be noted that VL is only one of the four knee extensor muscles and that the reported changes may not correspond to the behavior of the other three muscles. Although the fixation of the hip joint both in maximal and submaximal conditions limited the function of rectus femoris as a knee extensor only, caution should be taken when generalizing the results from the VL muscle to be valid for other quadriceps muscles with different intrinsic properties and geometry.

The model of Hawkins \& Hull (1990) was used to determine changes in the entire VL muscle-tendon unit. The model input requires joint angular movements, directly recorded with a potentiometer in the leg extension ergometer and obtained from a high-speed video analysis $(200 \mathrm{~Hz}$, Peak Performance Technologies, USA) in the sledge-jump performances. An electronic pulse was used to synchronize the analog and video data.

\section{Statistics}

Mean ( \pm S.E.M.) values are reported for 9 subjects. The differences between ISO and SSC conditions in maximum knee extension and between CMJ and REP in the sledge performances were tested using a two-tailed Student's t-test for paired samples. The significance level was set at $p<0.05$.

\section{Results}

In maximal knee extension the concentric torque production was enhanced when the shortening was preceded by stretch in SSC (Fig. 5). This was true even when the torque prior to the concentric phase was smaller $(p<0.05)$ in SSC than in ISO $(268 \pm 17 \mathrm{Nm}$ vs. $314 \pm 17 \mathrm{Nm}$, respectively). The torque was significantly higher in SSC than in ISO at the knee angle of $115^{\circ}(272 \pm 19 \mathrm{Nm}$ vs. $248 \pm 19$ $\mathrm{Nm}$, respectively) and the difference diminished when the knee was extended further (Fig. 6). Prior to and at the early phases of concentric action the EMG activities were not different (ns.). The EMG levels were equal when the force enhancement was significant at a knee angle of $115^{\circ}$. Figures 5 and 6 show that the VL fascicle was actively lengthened during the eccentric phase, being significantly longer at the beginning of shortening in SSC than in ISO (16.8 \pm 5.7 $\mathrm{cm}$ vs. $12.7 \pm 2.9 \mathrm{~cm})$. At the angle of $115^{\circ}$ the $2.1 \mathrm{~cm}$ difference in fascicle length 
$(14.4 \pm 3.7 \mathrm{~cm}$ vs. $12.3 \pm 3.3 \mathrm{~cm}$ in SSC and ISO, respectively) was not statistically significant. However, large inter-individual differences in fascicle length and low number of subjects must be acknowledged when these results are interpreted. During the concentric phase fascicles shortened $3.4 \mathrm{~cm}$ more $(p<$ 0.05) in SSC than in ISO. As knee angular movement was the same in SSC and ISO, also the changes in VL muscle-tendon unit length were equal.

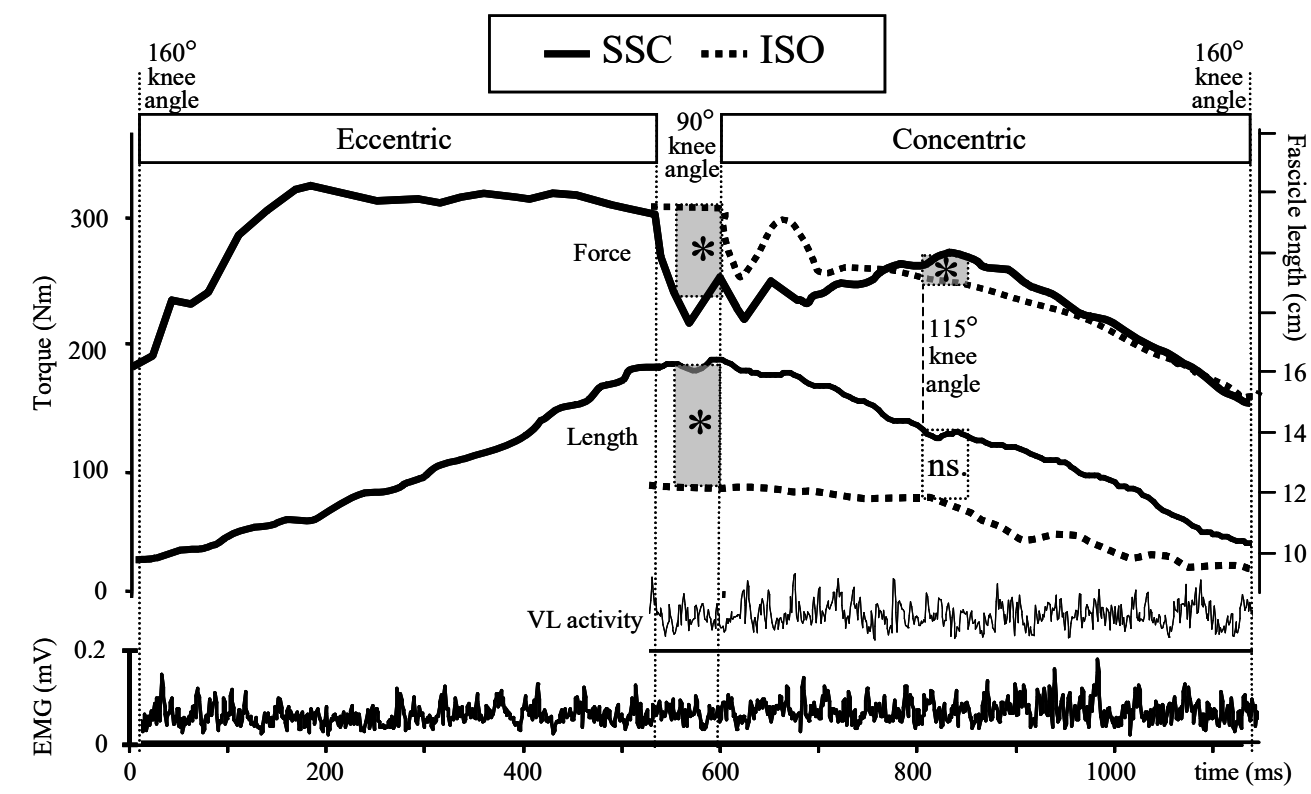

FIGURE 5 Knee extension torque (upper curves), fascicle lengths (middle curves) and rectified vastus lateralis EMG activity in SSC (solid lines) and ISO (dashed lines). During the eccentric phase in SSC the fascicles lengthened actively and the torque increased. During the subsequent $80 \mathrm{~ms}$ transition the torque dropped but fascicle length remained nearly constant. Consequently, the torque prior to the concentric phase was higher in ISO than in SSC. At a knee angle of $115^{\circ}$ the force was higher in SSC than in CON, but no significant differences in fascicle length or EMG were found. The shaded areas with stars indicate time periods where significant differences were found.
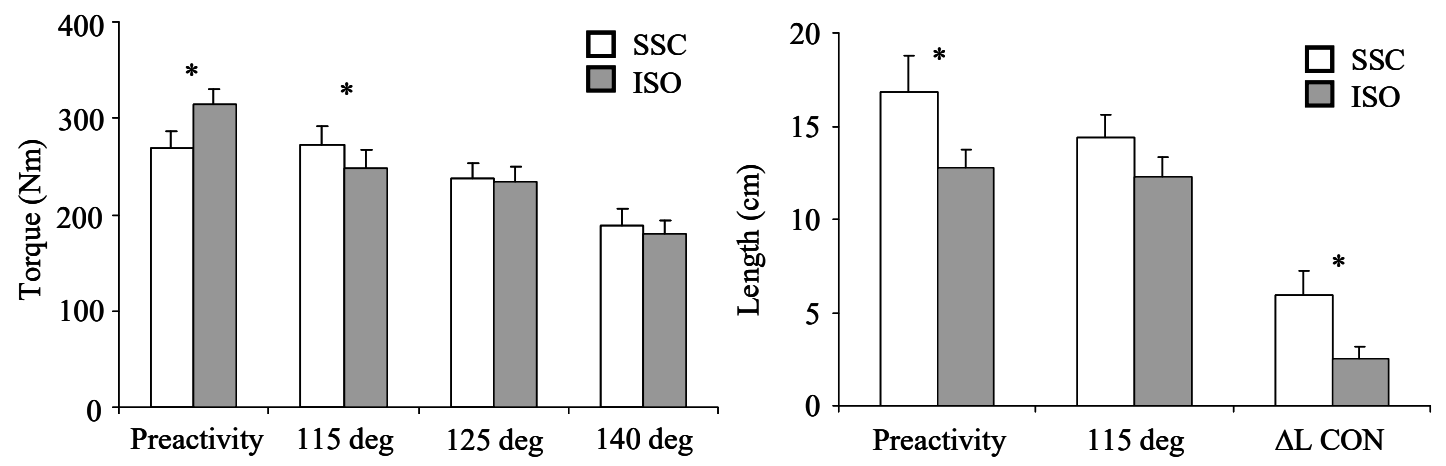

FIGURE 6 The knee extension torque (left) prior to concentric action was significantly higher $\left({ }^{*}, \mathrm{p}>0.05\right)$ with isometric pre-activation (ISO) as compared to the prestretch condition (SSC) at the knee angle of $90^{\circ}$. At the angle of $115^{\circ}$, the stretchinduced enhancement in the torque production was significant, but not at later phases of the movement. Fascicle length (right) prior to concentric action was higher in SSC and showed more pronounced shortening than in ISO. Error bars represent S.E.M. 
In submaximal sledge performances where the concentric net impulse was kept the same in REP and CMJ, the absolute contact times were $542 \pm 39 \mathrm{~ms}$ and $823 \pm 37$ $\mathrm{ms}$, respectively. The average $\mathrm{Fz}$ in the braking phase was $460 \mathrm{~N}$ greater $(p<$ 0.001) in REP than in CMJ (Fig. 7, 8). Similarly, aEMG of the knee extensor muscles in the braking phase was greater in REP than in CMJ, especially for vastus lateralis and medialis muscles. In the push-off phase aEMG levels did not differ significantly. Changes in the VL fascicle length were significantly smaller in REP than in CMJ both in the eccentric $(2.5 \pm 0.3$ vs. $3.5 \pm 0.5 \mathrm{~cm})$ and the concentric phase $(1.8 \pm 0.5$ vs. $3.3 \pm 0.5 \mathrm{~cm}$, respectively). However, the absolute difference in fascicle length at the end of the eccentric phase (longest lengths) was not significant between jumping conditions (9.3 \pm 0.6 vs. $10.9 \pm 1.4$ $\mathrm{cm}$ in CMJ and REP). Changes in VL muscle-tendon unit lengths were the same in both conditions (Fig. 7).

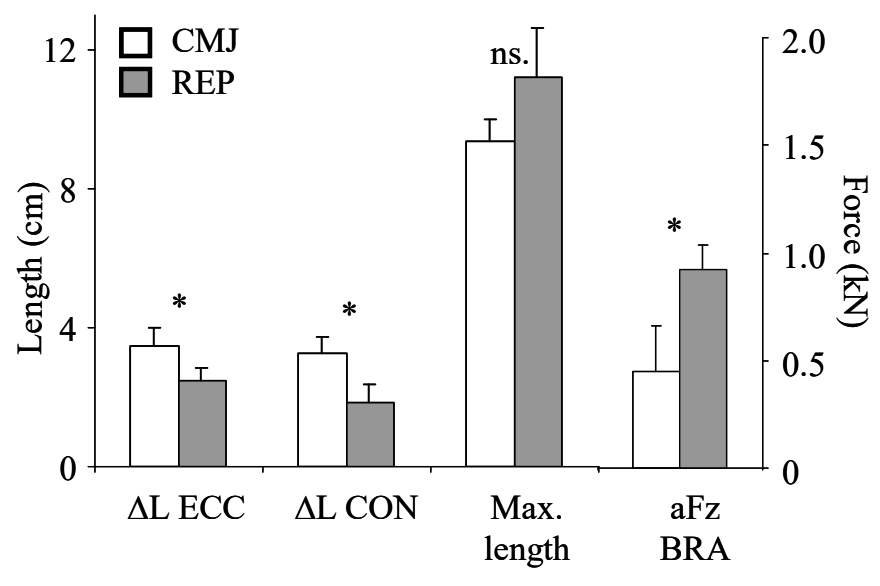

FIGURE 7 Changes in fascicle length in the eccentric phase ( $\triangle \mathrm{L}$ ECC) and the concentric phase $(\triangle \mathrm{L} C \mathrm{CON})$ and in the average reaction force in the braking phase $(\mathrm{aFz}$ BRA) were significant $(p<0.05)$ during submaximal sledge jumps. The maximal length values reached in the transition from lengthening to shortening were not different between CMJ and REP. Error bars represent S.E.M.

\section{Discussion}

The present study aimed to clarify the factors behind enhanced concentric performance during human movement. The results from isokinetic knee extension show that the force production was enhanced following a stretch even when the force prior to the concentric movement was smaller in SSC than in ISO. The fascicle length of the vastus lateralis muscle was longer in SSC than in ISO prior to the concentric phase but it did not differ between conditions at the time of force enhancement. Results from submaximal jumping performances show that in SSC exercises the increased pre-stretch load with greater eccentric EMG activity reduces changes in fascicle length. 


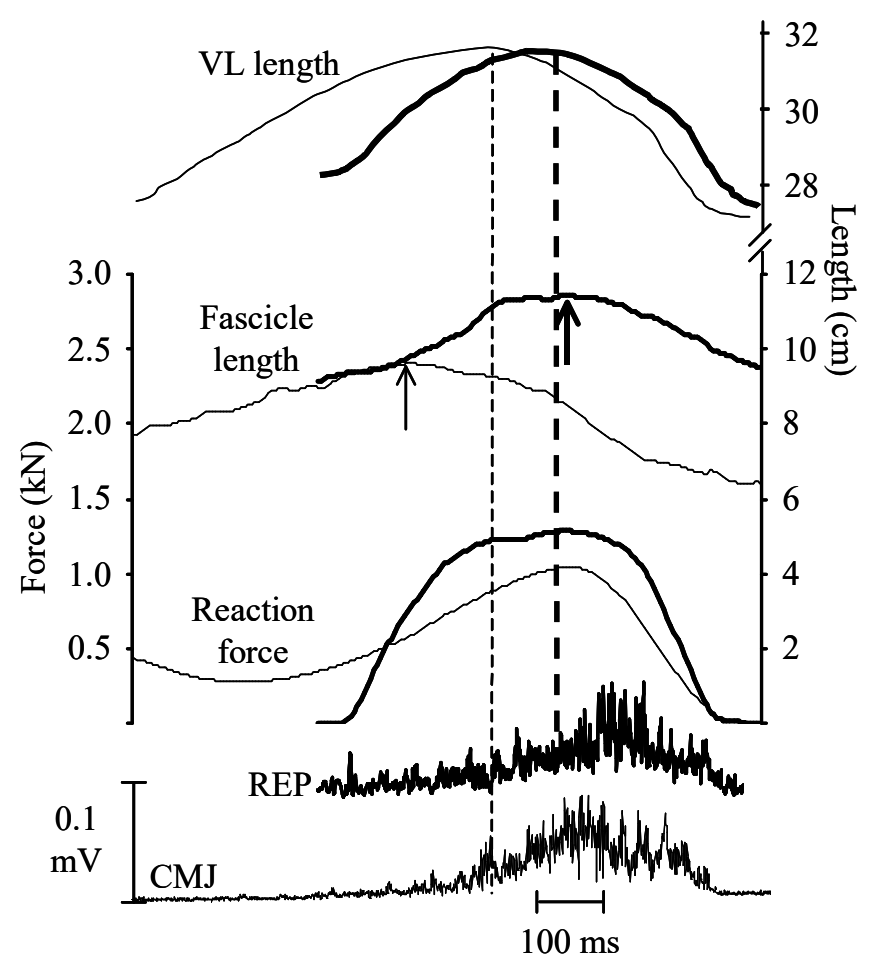

FIGURE 8 The time course of reaction forces, vastus lateralis EMG activity and the changes in fascicle and muscle-tendon length during a counter movement jump (CMJ, thin lines) and repeated drop jumps (REP, thick lines). The arrows and the vertical lines indicate transition from a lengthening phase to a shortening phase in the fascicle or in the muscle-tendon unit level, respectively. In CMJ the early shortening of fascicle is possible because of increasing EMG activity. Simultaneously, the force is still increasing and it lengthens the entire muscletendon unit. In REP the lengthening and shortening of the fascicle and the muscle-tendon unit occur in phase.

Normally when force enhancement has been reported, the experimental setting has allowed greater force prior to the concentric phase in the pre-stretch than in the pre-isometric condition (Cavagna et al. 1968, Takarada et al. 1997a,b, Komi 1983). This has been seen as an explanation of the phenomenon that occurs also during normal locomotion; for example, the greater jumping height in CMJ than in SJ has been seen as a result of a greater force level reached prior to the concentric phase (Bobbert et al. 1996). However, true as the observed relationship between the pre-force level and concentric output may be, the behaviour of the intact neuromuscular system may be more complex in its influence on muscle-tendon interaction. This may then have consequences in the concentric phase as well as in the economy of the entire performance. The following discussion attempts to point out some aspects that may explain the present results and their relevance to understanding the mechanisms behind potentiation associated with SSC muscle function in vivo. 


\section{Stretch-induced force enhancement}

First, it must be noted that in many animal studies the stretch-induced force enhancement phenomenon has been measured during the isometric phase following lengthening. This involves different contraction dynamics from the conditions of the present study where the phenomenon was examined during the concentric phase (Ettema et al. 1992). In addition, the results are influenced by differences in the amount of elastic compliance included in the examined muscle, which complicates the comparison of studies in different experimental conditions. Edman (1997) and Edman et al. (1978) have suggested that residual force enhancement, the long-lasting phenomenon present in the descending limb of the force-length relationship, could be an operative mechanism during locomotion in vivo. However, Brown \& Loeb (2000) conclude that the effects of the contractile component on force enhancement in an intact neuromuscular system are negligible. This can be understood from the fact that in-series compliance and angulation of fascicles in a pennate muscle induce a delay between the changes in the fascicle length and in the length of the entire muscle-tendon unit (Scott et al. 1996), and that the transient force enhancement decays rapidly $(\sim 20 \mathrm{~ms})$, requiring only small reverse changes in length (shortening) to abolish the phenomenon (Brown \& Loeb 2000).

It would seem odd if this force enhancement phenomenon in the contractile level existed without any effects on the force observed externally. If the contractile component is involved in stretch-induced force enhancement in vivo, only a very short delay between stretch and shortening can occur. This was clearly not the case in the present study; in SSC there was a delay of $80 \mathrm{~ms}$, and also in CMJ and REP the coupling time was quite long (see knee angular changes in Fig. 2). Another reason why stretch-induced residual force enhancement cannot be effective in all SSC exercises in vivo is that in submaximal CMJ, for example, the lengthening of the muscles is accompanied with very little EMG activity (Fig. 2, see also Finni et al. 2000).

\section{Force-length properties}

In more effective SSC exercises than CMJ, such as running, it has been demonstrated that by minimizing the changes in muscle length the tendon can gain and recoil energy while fibres perform very little work (Roberts et al. 1997). This behaviour can be considered economical in the light that eccentric and isometric loadings have been reported to require less EMG (Bigland-Ritchie \& Woods 1978, Komi 1973) or ATP (Ryschon et al. 1997) than a concentric one. The submaximal SSC exercises in the present study showed that an increase in prestretch intensity decreased the change in fascicle length (Fig.7). Although concentric aEMG was the same in both jumping conditions, a decreased activity level in REP and a consequent increase in efficiency could be expected if prestretch loads were further increased (Aura \& Komi 1986). 
During maximal voluntary knee extension the fascicle shortening was greater in SSC than in ISO (Figs. 5 and 6). In SSC, the fascicles were stretched to a longer length prior to concentric movement. Consequently, the tendinous tissue was longer in ISO than in SSC when the length of the entire muscle-tendon unit was equal in both conditions. During the $80 \mathrm{~ms}$ delay in SSC, the force decreased immediately when the movement stopped. As the fascicle length did not change, it is likely that the series elastic elements (SEE) shortened during this unloading. In ISO the rapid unloading of SEE occurred at the beginning of the concentric phase, delaying the initiation of fascicle shortening. It has been shown that during the shortening the decrease in muscle force depends on the initial muscle length and that the force depression is more pronounced in the ascending limb of the force-length relationship (Meijer et al. 1997). Longer initial length and the $80 \mathrm{~ms}$ delay in SSC may thus serve as potential explanatory factors for the force enhancement observed in the this study.

The force-length characteristics have been reported to change as a function of time during sustained isometric (see Huijing 1998) and lengthening contractions (Edman et al. 1978). In addition, because of a phase delay between varying muscle force and a consequent change in fascicle length, due to viscoelastic properties of tendon and aponeurosis, the force-length characteristics may contain velocity effects. These factors, including possible shift in force-length curve, may also be related to the potentiation observed during shortening in the SSC.

\section{In vivo muscle function}

In the present study, maximal voluntary activation in the knee extension exercises produced a force pattern that differs from the bouncing ball-type loading pattern observed in natural SSC exercises, characterized by varying activity (Komi et al. 1992). Thus, the SSC vs. ISO comparison of this study may not provide a correct explanation for the potentiation phenomenon which occurs in normal locomotion.

In our everyday locomotion, natural SSC muscle function is utilized with different intensities. Differences in the activation level may be a source for different functions that serve to economize the performance. In the light of our results, in CMJ the initial muscle force is produced when the muscle is first lengthened nearly passively (see Finni et al. 2000), requiring very little energy. If there is a demand for greater activity, as in REP-type jumps, the elastic energy that tendinous tissue can store and recoil will also have an effect on the performance, as seen in running turkeys (Roberts et al. 1997).

A contribution of reflex-induced force enhancement may also explain the potentiation phenomenon during natural locomotion (Komi 2000). In the present protocol, however, the prestretch load and velocity in both CMJ and 
REP were relatively low and a reflex contribution could not be identified (Fig. 2, 8) in contrast to higher intensity loading (Avela \& Komi 1998).

\section{Conclusions}

Concentric knee extension torque production during human movement was examined in various conditions. The purpose was to monitor factors that may play a role in enhancing performance in SSC exercises in vivo. The results from maximal isokinetic knee extension showed that the concentric torque production was enhanced after pre-stretch as compared to the pre-isometric condition. This enhancement may be related to modified length-tension properties together with longer initial fascicle length prior to shortening. Control of fascicle lengths was found to be important also in submaximal SSC exercise where a greater pre-load and eccentric activity prior to shortening decreased the changes in the fascicle length. This behaviour may be related to an energy savings mechanism; isometric contraction consumes less energy than the concentric one and simultaneously the tendon can act as a spring storing and recoiling elastic energy.

In general, muscular output depends on the interaction between muscle and tendon lengths with a given force, activity level, length and velocity. In this regard human subjects may show individual activity patterns and thus also several patterns of behaviour may be found during natural movements. For future research, electrical stimulation could be used to minimize the differences in neural activity and thus examine how the possible structural and functional differences affect muscle function in vivo.

\section{Acknowledgements}

The authors express their gratitude to the LIKES Research Center for collaboration. This study was supported by a grant $(119 / 7222 / 99)$ from the Ministry of Education, Finland. 


\section{References}

Asmussen, E. \& Bonde-Petersen, F. 1974 Apparent efficiency and storage of elastic energy in human muscles during exercise. Acta Physiol Scand 92, 537-545.

Aura, O. \& Komi, P.V. 1986 The mechanical efficiency of locomotion in men and women with special emphasis on stretch-shortening cycle exercises. Eur J Appl Physiol 55, 37-43.

Avela, J. \& Komi, P.V. 1998 Reduced stretch reflex sensitivity and muscle stiffness after long-lasting stretch-shortening cycle exercise in humans. Eur J Appl Physiol 78, 403-410.

Bigland-Richie, B. \& Woods, J.J. 1976 Integrated electromyogram and oxygen uptake during positive and negative work. J Physiol 260, 267-277.

Bobbert, M.F., Gerritsen, K.G.M., Litjens, M.C.A. \& van Soest, A.J. 1996 Why is countermovement jump height greater than squat jump height? Med Sci Sports Exerc 28, 1402-1412.

Bosco, C., Viitasalo, J.T., Komi, P.V. \& Luhtanen, P. 1982 Combined effects of elastic energy and myoelectrical potentiation during stretch-shortening cycle exercise. Acta Physiol Scand 114, 557- 565.

Bosco, C., Komi, P.V. \& Ito, A. 1981 Prestretch potentiation of human skeletal muscle during ballistic movement. Acta Physiol Scand 114, 557- 565.

Brown, I.E., \& Loeb, G.E. 2000 Measured and modeled properties of mammalian skeletal muscle: III. the effects of stimulus frequency on stretch-induced force enhancement and shortening- induced force depression. J Muscle Res Cell Motil 21, 21-31.

Cavagna, G.A., Dusman, B. \& Margaria, R. 1968 Positive work done by a previously stretched muscle. J Appl Physiol 1, 21-32.

Edman, K.P.A. 1997 Force enhancement by stretch. J Appl Biomech 13, 389-415.

Edman, K.P.A., Elzinga, G. \& Noble, M.I.M. 1978 Enhancement of mechanical performance by stretch during tetanic contractions of vertebrate skeletal muscle fibres. J Physiol 281, 139-155.

Ettema, G.J.C., Huijing, P.A. \& De Haan, A. 1992 The potentiating effect of prestretch on the contractile performance of rat gastrocnemius medialis muscle during subsequent shortening and isometric contractions. J Exp Biol 165, 121-136.

Finni, T., Komi, P.V. \& Lepola, V. 2000 In vivo triceps surae and quadriceps femoris muscle function in a squat jump and counter movement jump. Eur J Appl Physiol 83, 416-426. 
Finni, T., Ikegawa, S., Lepola, V. \& Komi, P.V. 2001 In vivo behavior of vastus lateralis muscle during dynamic performance. Eur J Sport Sci.1,1.

Gollhofer, A., Strojnik, V., Rapp, W. \& Schweizer, L. 1992 Behaviour of triceps surae muscle-tendon complex in different conditions. Eur J Appl Physiol 64, 283-291.

Hawkins, D. \& Hull, M.L. 1990 A method for determining lower extremity muscle-tendon lengths during flexion/extension movements. J Biomech 5, 487-494.

Herzog, W. \& Leonard, T.R. 2000 The history dependence of force production in mammalian skeletal muscle following stretch-shortening and shortening-stretch cycles. J Biomech 33, 531-542.

Huijing, P.A. 1998 Muscle, the motor of movement: properties in function, experiment and modelling. J Electromyogr Kinesiol 8, 61-77.

Ichinose, Y., Kawakami, Y., Ito, M. \& Fukunaga, T. 1997 Estimation of active force-length characteristics of human vastus lateralis muscle. Acta Anat 159, 78-83.

Komi, P.V. (1973) Relationship between muscle, tendon, EMG and velocity of contraction under concentric and eccentric work. In: J. E. Desmendt (ed.) New Developments in Electromyoraphy and Clinical Neurophysiology, vol. 1, pp. 596-606. Karger, Basel.

Komi, P.V. 1983 Elastic potentiation of muscle and its influence on sport performance. In: Baumann W. (ed.) Biomechanics and Performance in Sport p. 61. Verlag Karl Hofmann Schorndorf, Germany.

Komi, P.V. 2000 Stretch-shortening cycle: a powerful model to study normal and fatigued muscle. J Biomech 33, 1197-1206.

Komi, P.V., Fukashiro, M.S. \& Järvinen, M. (1992) Biomechanical loading of Achilles tendon during normal locomotion. Clin Sport Med 11, 3, 521-531

Komi, P.V. \& Gollhofer, A. (1997) Stretch reflexes can have an important role in force enhancement during SSC exercise. J Appl Biomech 13, 4, 451-460.

Komi, P.V., Linnamo, V., Silventoinen, P. \& Sillanpää, M. 2000 Force and EMG power spectrum during eccentric and concentric actions. Med Sci Sports Exerc 32, 10, 1757-1762.

Kyröläinen, H. \& Komi, P.V. 1995 Differences in mechanical efficiency between power-and endurance-trained athletes while jumping. Eur J Appl Physiol 70, 36-44.

Meijer, K., Grootenboer, H.J., Koopman, B.F.J.M. \& Huijing, P.A. 1997 Fully isometric length-force curves of rat muscle differ from those during and after concentric contractions. J Appl Biomech 13, 164-181. 
Norman, R.W. \& Komi, P.V. (1979) Electromechanical delay in skeletal muscle under normal movement conditions. Acta Physiol Scand 106, 241-248.

Roberts, T.J., Marsh, R.L., Weyand, P.G. \& Taylor, C.R. 1997 Muscular force in running turkeys: the economy of minimizing work. Science 21, 1113-1115.

Ryschon, T.W., Fowler, M.D., Wysong, R.E., Anthony, A.-R. \& Balaban, R.S. 1997 Efficiency of human skeletal muscle in vivo: comparison of isometric, concentric, and eccentric muscle action. J Appl Physiol 83, 867-874.

Scott, S.H., Brown, I.E. \& Loeb, G.E. 1996 Mechanics of feline soleus: I. Effect of fascicle length and velocity on force output. J Muscle Res Cell Mot 17, 207-219.

Takarada, Y., Hirano, Y., Ishige, Y. \& Ishii, N. 1997a Stretch-induced enhancement of mechanical power output in human multijoint exercise with countermovement. J Appl Physiol 83, 1749-1755.

Takarada, Y., Iwamoto, H., Sugi, H., Hirano, Y. \& Ishii, N. 1997b Stretch-induced enhancement of mechanical work production in frog single fibers and human muscle. $J$ Appl Physiol 83, 1741-1748. 


\section{Figure legends}

Fig. 1. Experimental conditions in the maximal knee extension with isometric-concentric (ISO) and eccentric-concentric (SSC) muscle action. Average torque-time curves are shown in the upper curve, divided into different phases according to the changes in the knee joint angle (2. curve from top). Numbered phases between vertical lines correspond to 1) maximal isometric loading (note: different starting angles in ISO and SSC), 2) lengthening and 3) shortening of quadriceps femoris muscles. The delay between the phases 2 and 3 in SSC is caused by the properties of the device. The shortening phase (3) was divided into $50 \mathrm{~ms}$ phases and average force and EMG values were calculated during those periods. Rectified EMG recordings $(\mathrm{n}=9)$ from biceps femoris $(\mathrm{BF})$, rectus femoris $(\mathrm{RF})$, vastus medialis $(\mathrm{VM})$ and vastus lateralis (VL) showed no marked differences during the concentric phase. The initial torque rise is dashed schematically in the figure. The group mean EMG recordings for this period could not be obtained. The filled circles represent mean torques measured during maximal voluntary contraction at the given knee angle.

Fig. 2. Reaction force (upper), knee joint angle (middle) and rectified EMG recordings during submaximal, unilateral counter movement jump (CMJ) and repeated drop jump (REP). For other definitions, see Fig. 1.

Fig. 3 An example of raw data recordings from repeated drop jumps. The curves show changes in vastus lateralis muscle-tendon length (upper), fascicle length (lowest curve) and reaction force (dashed line).

Fig. 4. The model used for fascicle length determination. The fascicle length $\left(l_{1}\right)$ measured from the image was added to the estimated length $\left[\mathrm{h}(\sin \beta)^{-1}\right]$ to obtain the total fascicle length. The ending points of the chosen $l_{1}$ were tracked throughout the motion. In the model the spatial coordinates of the ending points of the measure $h$ are fixed horizontally to the right end of the visualized area but not in respect to its vertical position or to its position in respect to the fascicle.

Fig. 5. Knee extension torque (upper curves), fascicle lengths (middle curves) and rectified vastus lateralis EMG activity in SSC (solid lines) and ISO (dashed lines). During the eccentric phase in SSC the fascicles lengthened actively and the torque increased. During the subsequent $80 \mathrm{~ms}$ transition the torque dropped but fascicle length remained nearly constant. Consequently, the torque prior to the concentric phase was higher in ISO than in SSC. At a knee angle of $115^{\circ}$ the force was higher in SSC than in CON, but no significant differences in fascicle length or EMG were found. The shaded areas with stars indicate time periods where significant differences were found.

Fig. 6. The knee extension torque (a) prior to concentric action was significantly higher $\left({ }^{*}, \mathrm{p}>0.05\right)$ with isometric pre-activation (ISO) as compared to the pre-stretch condition (SSC) at the knee angle of $90^{\circ}$. At the angle of $115^{\circ}$, the stretch-induced enhancement in the torque production was significant, but not at later phases of the movement. Fascicle length (b) prior to concentric action was higher in SSC and showed more pronounced shortening than in ISO. Error bars represent S.E.M..

Fig. 7. Changes in fascicle length in the eccentric phase ( $\triangle \mathrm{L}$ ECC) and the concentric phase $(\Delta \mathrm{L} C \mathrm{CON})$ and in the average reaction force in the braking phase $(\mathrm{aFz} B R A)$ were significant $(p<0.05)$ during submaximal sledge jumps. The maximal length values reached in the transition from lengthening to shortening were not different between CMJ and REP. Error bars represent S.E.M.

Fig. 8. The time course of reaction forces, vastus lateralis EMG activity and the changes in fascicle and muscle-tendon length during a counter movement jump (CMJ, thin lines) and repeated drop jumps (REP, thick lines). The arrows and the vertical lines indicate transition from a lengthening phase to a shortening phase in the fascicle or in the muscle-tendon unit level, respectively. In CMJ the early 
shortening of fascicle is possible because of increasing EMG activity. Simultaneously, the force is still increasing and it lengthens the entire muscle-tendon unit. In REP the lengthening and shortening of the fascicle and the muscle-tendon unit occur in phase. 